\title{
NOTE ON THE CHARACTERISTIC RANK OF VECTOR BUNDLES
}

\author{
ANIRUDDHA C. NAOLEKAR AND AJAY SINGH THAKUR
}

\begin{abstract}
We define the notion of characteristic $\operatorname{rank}$, $\operatorname{charrank}_{X}(\xi)$, of a real vector bundle $\xi$ over a connected finite $C W$-complex $X$. This is a bundle-dependent version of the notion of characteristic rank introduced by Július Korbaš in 2010. We obtain bounds for the cup length of manifolds in terms of the characteristic rank of vector bundles generalizing a theorem of Korbaš and compute the characteristic rank of vector bundles over the Dold manifolds, the Moore spaces and the stunted projective spaces amongst others.
\end{abstract}

\section{INTRODUCTION}

Recently, J. Korbaš [10] has introduced a new homotopy invariant, called the characteristic rank, of a connected closed smooth manifold $X$. The characteristic rank of a connected closed smooth $d$-manifold $X$, denoted by charrank $(X)$, is the largest integer $k, 0 \leq k \leq d$, such that every cohomology class $x \in H^{j}\left(X ; \mathbb{Z}_{2}\right)$, $0 \leq j \leq k$ is a polynomial in the Stiefel-Whitney classes of (the tangent bundle of) $X$.

Apart from being an interesting question in its own right, part of the motivation for computing the characteristic rank comes from a theorem of Korbaš ([10], Theorem 1.1), where the author has described a bound for the $\mathbb{Z}_{2}$-cuplength of (unorientedly) null cobordant closed smooth manifolds in terms of their charateristic rank. More specifically, Korbaš has proved the following.

Theorem 1.1. ([10], Theorem 1.1) Let $X$ be a closed smooth connected $d$ dimensional manifold unorientedly cobordant to zero. Let $\widetilde{H}^{r}\left(X ; \mathbb{Z}_{2}\right), r<d$, be the first nonzero reduced cohomology group of $X$. Let $z(z<d-1)$ be an integer such that for $j \leq z$ each element of $H^{j}\left(X ; \mathbb{Z}_{2}\right)$ can be expressed as a polynomial in the Stiefel-Whitney classes of the manifold $X$. Then we have that

$$
\operatorname{cup}(X) \leq 1+\frac{d-z-1}{r} \text {. }
$$

2010 Mathematics Subject Classification. 57R20.

Key words and phrases. Stiefel-Whitney class, characteristic rank, Dold manifold, Moore space, stunted projective space. 
Recall that the $\mathbb{Z}_{2}$-cup-length, denoted by $\operatorname{cup}(X)$, of a space $X$ is the largest integer $t$ such that there exist classes $x_{i} \in H^{*}\left(X ; \mathbb{Z}_{2}\right), \operatorname{deg}\left(x_{i}\right) \geq 1$, such that the cup product $x_{1} \cdot x_{2} \cdots x_{t} \neq 0$. We mention in passing that the $\mathbb{Z}_{2}$-cup-length is well known to have connections with the Lyusternik-Shnirel'man category of the space.

With the computation of the characteristic rank in mind, Balko and Korbaš 3 obtained bounds for the characteristic rank of manifolds $X$ which occur as total spaces of smooth fiber bundles with fibers totally non-homologous to zero, and also in the situation where, additionally, $X$ itself is null cobordant (see [3], Theorems 2.1 and 2.2).

It is useful to think of the characteristic rank of a manifold as the characteristic rank "with respect to the tangent bundle" and introduce bundle-dependency as in the definition below.

Definition 1.1. Let $X$ be a connected, finite $C W$-complex and $\xi$ a real vector bundle over $X$. The characteristic rank of the vector bundle $\xi$ over $X$, denoted by $\operatorname{charrank}_{X}(\xi)$, is by definition the largest integer $k, 0 \leq k \leq \operatorname{dim}(X)$, such that every cohomology class $x \in H^{j}\left(X ; \mathbb{Z}_{2}\right), 0 \leq j \leq k$, is a polynomial in the Stiefel-Whitney classes $w_{i}(\xi)$ of $\xi$. The upper characteristic rank of $X$, denoted by ucharrank $(X)$, is the maximum of $\operatorname{charrank}_{X}(\xi)$ as $\xi$ varies over all vector bundles over $X$.

Thus, if $X$ is a connected closed smooth manifold, then $\operatorname{charrank}_{X}(T X)=$ $\operatorname{charrank}(X)$ where $T X$ is the tangent bundle of $X$. Note that if $X$ and $Y$ are homotopically equivalent closed connected smooth manifolds, then ucharrank $(X)=$ ucharrank $(Y)$.

In this note we discuss some general properties of $\operatorname{charrank}(\xi)$ and give a complete description of $\operatorname{charrank}_{X}(\xi)$ of vector bundles $\xi$ over $X$ when $X$ is: a product of spheres, the real and complex projective spaces, the Dold manifold $P(m, n)$, the Moore space $M\left(\mathbb{Z}_{2}, n\right)$ and the stunted projective spaces $\mathbb{R} \mathbb{P}^{n} / \mathbb{R P}^{m}$. We now briefly describe the contents of this note.

For a connected finite $C W$-complex $X$, let $r_{X}$ denote the smallest positive integer such that $\widetilde{H}^{r_{X}}\left(X ; \mathbb{Z}_{2}\right) \neq 0$. In the case that such an integer does not exist, that is, all the reduced cohomology groups $\widetilde{H}^{i}\left(X ; \mathbb{Z}_{2}\right)=0,1 \leq i \leq$ $\operatorname{dim}(X)$, we set $r_{X}=\operatorname{dim}(X)+1$. In any case, $r_{X} \geq 1$.

Making the definition of the characteristic rank bundle-dependent gives the following theorem which is a straighforward generalisation of Theorem[1.1. In this form the theorem yields sharper bounds on the cup-length in certain cases (see Examples 3 and 4 below). We shall prove the following.

Theorem 1.2. Let $X$ be a connected closed smooth d-manifold. Let $\xi$ be a vector bundle over $X$ satisfying the following: 
- there exists $k, k \leq \operatorname{charrank}_{X}(\xi)$, such that every monomial

$$
w_{i_{1}}(\xi) \cdots w_{i_{r}}(\xi), 0 \leq i_{t} \leq k
$$

of total degree $d$ is zero.

Then,

$$
\operatorname{cup}(X) \leq 1+\frac{d-k-1}{r_{X}}
$$

We note that if $X$ is an unoriented boundary, then $\xi=T X$ satisfies the conditions of the theorem above with $k=\operatorname{charrank}_{X}(T X)$. In this theorem we do not assume that $X$ is an unoriented boundary.

If $X$ is an unoriented boundary and there exists a vector bundle $\xi$ over $X$ with $k$ satisfying the conditions of the above theorem, such that

$$
\operatorname{charrank}(X)=\operatorname{charrank}_{X}(T X)<k \leq \operatorname{charrank}_{X}(\xi),
$$

then the bound for $\operatorname{cup}(X)$ using $k$ is sharper than that obtained from Theorem 1.1. We note that over the null cobordant manifold $S^{d} \times S^{m}, d=2,4,8$, and $m \neq 2,4,8$, there exists a vector bundle $\xi$ and an integer $k$ satisfying the conditions of Theorem 1.2 and equation 1.0 .1 (see Examples 3 , 4 below).

If $X$ is a connected closed smooth manifold with ucharrank $(X)=\operatorname{dim}(X)$, it turns out that the cup-length $\operatorname{cup}(X)$ of $X$ can be computed as the maximal length of a non-zero product of the Stiefel-Whitney classes of a suitable bundle over $X$. We prove the following.

Theorem 1.3. Let $X$ be a connected closed smooth d-manifold. If

$$
\operatorname{ucharrank}(X)=\operatorname{dim}(X) \text {, }
$$

then there exists a vector bundle $\xi$ over $X$ such that

$$
\operatorname{cup}(X)=\max \left\{k \mid \text { there exist } i_{1}, \ldots, i_{k} \geq 1 \text { with } w_{i_{1}}(\xi) \cdots w_{i_{k}}(\xi) \neq 0\right\} .
$$

Making the definition of characteristic rank bundle-dependent allows us, under certain conditions, to construct an epimorphism $\widetilde{K O}(X) \longrightarrow \mathbb{Z}_{2}$. It is clear from the definition that $\operatorname{charrank}_{X}(\xi)=\operatorname{charrank}_{X}(\eta)$ if $\xi$ and $\eta$ are (stably) isomorphic. Let $\operatorname{Vect}_{\mathbb{R}}(X)$ denote the semi-ring of isomorphism classes of real vector bundles over $X$. We then have a function

$$
f: \operatorname{Vect}_{\mathbb{R}}(X) \longrightarrow \mathbb{Z}_{2}
$$

defined by $f(\xi)=\operatorname{charrank}_{X}(\xi)(\bmod 2)$. We observe that under certain restrictions on the values of $\operatorname{charrank}_{X}(\xi)$ the function $f$ is actually a semi-group homomorphism. More precisely we prove the following.

Theorem 1.4. Let $X$ be a connected finite $C W$-complex with $r_{X}=1$. Assume that for any vector bundle $\xi$ over $X$, $\operatorname{charrank}_{X}(\xi)$ is either $r_{X}-1=0$ or an odd integer. Assume that $\operatorname{ucharrank}(X) \geq 1$. Then the function

$$
f: \operatorname{Vect}_{\mathbb{R}}(X) \longrightarrow \mathbb{Z}_{2}
$$


defined by $f(\xi)=\operatorname{charrank}_{X}(\xi)(\bmod 2)$ is a surjective semi-group homomorphism and hence gives rise to a surjective group homomorphism $\tilde{f}: K O(X) \longrightarrow$ $\mathbb{Z}_{2}$. Furthermore, this restricts to an epimorphism $\tilde{f}: \widetilde{K O}(X) \longrightarrow \mathbb{Z}_{2}$.

The function $f$ defined in the theorem above is in general not a semi-ring homomorphism (see Remark3). There is a large class of spaces that satisfy the conditions of this theorem. We prove the following.

Theorem 1.5. (1) Let $X=\mathbb{R P}^{n}$. Then ucharrank $(X)=n$ and for any vector bundle $\xi$ over $X$, the characteristic rank $\operatorname{charrank}_{X}(\xi)$ is either $r_{X}-1=0$ or is $n$.

(2) Let $X=S^{1} \times \mathbb{C P}^{n}$. Then ucharrank $(X)=2 n+1$ and for any vector bundle $\xi$ over $X$, the characteristic rank charrank $_{X}(\xi)$ either is $r_{X}-1=$ 0,1 or $2 n+1$.

(3) Let $X$ be the Dold manifold $P(m, n)$. Then ucharrank $(X)=2 n+m$ and for any vector bundle $\xi$ over $X$, the characteristic rank $\operatorname{charrank}_{X}(\xi)$ is either $r_{X}-1=0,1$ or $2 n+m$.

Recall that the Dold manifold $P(m, n)$ is the quotient of $S^{m} \times \mathbb{C P}^{n}$ by the fixed point free involution $(x, z) \mapsto(-x, \bar{z})$.

In this note we concentrate on the computational part of characteristic rank of vector bundles. We compute the characteristic rank of vector bundles over products of spheres $S^{d} \times S^{m}$, the real and complex projective spaces, the spaces $S^{1} \times \mathbb{C P}^{n}$, the Dold manifold $P(m, n)$, the Moore space $M\left(\mathbb{Z}_{2}, n\right)$ and the stunted projective space $\mathbb{R} \mathbb{P}^{n} / \mathbb{R} \mathbb{P}^{m}$. We also prove some general facts about characteristic rank of vector bundles.

The paper is organized as follows. In Section 2 we prove some general facts about $\operatorname{charrank}(\xi)$. In Section 3 we prove Theorems1.2, 1.3 and 1.4. Finally, in Section 4, we compute $\operatorname{charrank}_{X}(\xi)$ where $X$ is one of the following spaces: the product of spheres $S^{d} \times S^{m}$, the real and complex projective spaces, the product $S^{1} \times \mathbb{C P}^{n}$, the Dold manifold $P(m, n)$, the Moore space $M\left(\mathbb{Z}_{2}, n\right)$ and the stunted projective space.

Convention. By a space we shall mean a connected finite $C W$-complex. All vector bundles are real unless otherwise stated.

\section{Generalities}

In this section we make some general observations about $\operatorname{charrank}(\xi)$. Recall that, for a space $X, r_{X}$ denotes the smallest positive integer for which the reduced cohomology group $\widetilde{H}^{r_{X}}\left(X ; \mathbb{Z}_{2}\right) \neq 0$, and if such an $r_{X}$ does not exist, then we set $r_{X}=\operatorname{dim}(X)+1$. Then for any vector bundle $\xi$ over $X$ we have

$$
r_{X}-1 \leq \operatorname{charrank}_{X}(\xi) \leq \operatorname{ucharrank}(X) .
$$

We begin with some easy observations. 
Lemma 2.1. Let $\xi$ and $\eta$ be any two vector bundles over a space $X$.

(1) If $w_{r_{X}}(\xi)=0$, then $\operatorname{charrank}_{X}(\xi)=r_{X}-1$;

(2) If $w(\xi)=1$, then $\operatorname{charrank}_{X}(\xi)=r_{X}-1$.

(3) If $w(\eta)=1$, then $\operatorname{charrank}_{X}(\xi \oplus \eta)=\operatorname{charrank}_{X}(\xi)$. Hence if $\widetilde{K O}(X)=$ 0 , then charrank $_{X}(\xi)=r_{X}-1$ for any vector bundle over $X$;

(4) If $\xi$ and $\eta$ are stably isomorphic, then $\operatorname{charrank}_{X}(\xi)=\operatorname{charrank}_{X}(\eta)$;

(5) There exists a vector bundle $\theta$ over $X$ such that $\operatorname{charrank}_{X}(\xi \oplus \theta)=$ $r_{X}-1$.

Proof. (1) follows from the definition. Clearly, (2) follows from (1). To prove (3) we note that since $w(\xi \oplus \eta)=w(\xi)$, we have $\operatorname{charrank}_{X}(\xi \oplus \eta)=\operatorname{charrank}_{X}(\xi)$. As $\widetilde{K O}(X)=0$, we have $\xi \oplus \varepsilon \cong \varepsilon^{\prime}$. Hence

$$
\operatorname{charrank}_{X}(\xi)=\operatorname{charrank}_{X}(\xi \oplus \varepsilon)=\operatorname{charrank}_{X}\left(\varepsilon^{\prime}\right)=r_{X}-1 .
$$

This completes the proof of (3). Next, if $\xi$ and $\eta$ are stably isomorphic, we have $\xi \oplus \varepsilon \cong \eta \oplus \varepsilon^{\prime}$ where $\varepsilon$ and $\varepsilon^{\prime}$ are trivial vector bundles. Hence (4) follows from (3). Finally, as $X$ is compact, given $\xi$ we can find a vector bundle $\theta$ such that $\xi \oplus \theta \cong \varepsilon$. Hence (5) follows from (4) and (2).

Lemma 2.2. Let $X$ be a space and $1 \leq r_{X} \leq \operatorname{dim}(X)$

(1) If ucharrank $(X) \geq r_{X}$, then $\operatorname{dim}_{\mathbb{Z}_{2}} H^{r_{X}}\left(X ; \mathbb{Z}_{2}\right)=1$.

(2) If $r_{X}$ is not a power of 2 , then $\mathrm{ucharrank}(X)=r_{X}-1$.

Proof. If $\xi$ is a vector bundle over $X$ with $\operatorname{charrank}_{X}(\xi) \geq r_{X}$, then by Lemma2.1 $(1), w_{r_{X}}(\xi) \neq 0$. This forces the equality $\operatorname{dim}_{\mathbb{Z}_{2}} H^{r_{X}}\left(X ; \mathbb{Z}_{2}\right)=1$ and proves (1). It is known that for any vector bundle $\xi$, the smallest integer $k$ such that $w_{k}(\xi) \neq 0$ is always a power of 2 (see, for example, [12, page 94). Lemma2.1] (1) now completes the proof of (2).

Let $Y$ be a space and and let $X=\Sigma Y$ be the suspension of $Y$. Then any cup-product of elements of positive degree in $H^{*}\left(X ; \mathbb{Z}_{2}\right)$ is zero. The following lemma is an easy consequence of this fact and we omit the proof.

Lemma 2.3. Let $Y$ be a space and $X=\Sigma Y$. Let $k_{X}$ be an integer defined by

$$
k_{X}=\max \left\{k \mid \operatorname{dim}_{\mathbb{Z}_{2}} H^{j}\left(X ; \mathbb{Z}_{2}\right) \leq 1,0 \leq j \leq k, k \leq \operatorname{dim}(X)\right\} .
$$

Let $\xi$ be any vector bundle over $X$. Then, $\operatorname{charrank}_{X}(\xi) \leq k_{X}$. In particular, $\operatorname{ucharrank}(X) \leq k_{X}$.

Lemma 2.4. Let $f: X \longrightarrow Y$ be a map between spaces. If $f^{*}: H^{*}\left(Y ; \mathbb{Z}_{2}\right) \longrightarrow$ $H^{*}\left(X ; \mathbb{Z}_{2}\right)$ is surjective, then

$$
\operatorname{charrank}_{X}\left(f^{*} \xi\right) \geq \min \left\{\operatorname{charrank}_{Y}(\xi), \operatorname{dim}(X)\right\}
$$

for any vector bundle $\xi$ over $Y$. 
Proof. As $w_{i}\left(f^{*} \xi\right)=f^{*}\left(w_{i}(\xi)\right)$, the surjectivity of $f^{*}$ implies that every cohomology class in $H^{*}\left(X ; \mathbb{Z}_{2}\right)$ of degree at most $\operatorname{charrank}_{Y}(\xi)$ is a polynomial in the Stiefel-Whitney classes of $f^{*} \xi$. If $\operatorname{charrank}_{Y}(\xi) \geq \operatorname{dim}(X)$, then

$$
\operatorname{charrank}_{X}\left(f^{*} \xi\right)=\operatorname{dim}(X) \text {. }
$$

If $\operatorname{charrank}_{Y}(\xi) \leq \operatorname{dim}(X)$, then $\operatorname{charrank}_{Y}(\xi) \leq \operatorname{charrank}_{X}\left(f^{*} \xi\right) \leq \operatorname{dim}(X)$.

Before mentioning further general properties of the characteristic rank we record the characteristic rank of vector bundles over the sphere. The description of the characteristic rank of vector bundles over the spheres is an easy consequence of the following theorem due to Atiyah-Hirzebruch ([2], Theorem 1), (see also [1]).

Theorem 2.1. (2, Theorem 1) There exists a real vector bundle $\xi$ over the sphere $S^{d}$ with $w_{d}(\xi) \neq 0$ only for $d=1,2,4$, or 8 .

For the Hopf bundle $\nu_{d}$ over $S^{d}(d=1,2,4,8)$, the Stiefel-Whitney class $w_{d}\left(\nu_{d}\right)$ is not zero. Thus,

$$
\operatorname{ucharrank}\left(S^{d}\right)=\left\{\begin{array}{cl}
d & \text { if } d=1,2,4, \text { or } 8 \\
d-1 & \text { otherwise. }
\end{array}\right.
$$

Note that $\operatorname{charrank}\left(S^{d}\right)=d-1$. We shall use the above description of characteristic rank of vector bundles over the spheres in the sequel without explicit reference.

Suppose that $\pi: S^{d} \longrightarrow X$ is a $k$-sheeted covering with $k>1$ odd. Since $X \cong S^{d} / G$, where $G$ is a finite group with $|G|=k$, we have that $d$ is odd. By Proposition 3G.1 of [7, the homomorphism $\pi^{*}: H^{i}\left(X ; \mathbb{Z}_{2}\right) \longrightarrow H^{i}\left(S^{d} ; \mathbb{Z}_{2}\right)$ is a monomorphism with image the $G$-invariant elements for all $i \geq 0$. In particular, $H^{i}\left(X ; \mathbb{Z}_{2}\right)=0,0<i<d$ and $\pi^{*}: H^{d}\left(X ; \mathbb{Z}_{2}\right) \longrightarrow H^{d}\left(S^{d} ; \mathbb{Z}_{2}\right) \cong \mathbb{Z}_{2}$ is an isomorphism. Thus we have the following corollary to Theorem 2.1

Corollary 2.1.1. Assume that $\pi: S^{d} \longrightarrow X$ is a k-sheeted covering with an odd $k>1$ and $d \neq 1$. Then $w(\xi)=1$ for any vector bundle $\xi$ over $X$ and we have $\operatorname{ucharrank}(X)=d-1$.

Proof. If $0<i<d$, then obviously $w_{i}(\xi)=0$. In addition, for any $\xi$ we have now $\pi^{*}\left(w_{d}(\xi)\right)=w_{d}\left(\pi^{*} \xi\right)=0$ by Theorem 2.1. Since, $\pi^{*}$ is injective, we thus have $w_{d}(\xi)=0$. We know that $H^{d}\left(X, \mathbb{Z}_{2}\right) \cong \mathbb{Z}_{2}$; this implies that $\operatorname{charrank}_{X}(\xi) \leq d-1$ for any $\xi$. The inequality $\operatorname{charrank}_{X}(\xi) \geq d-1$ for any $\xi$ is clear.

Example 1. Let $L=L_{m}\left(\ell_{1}, \ldots, \ell_{n}\right)$ denote the lens space which is a quotient of $S^{2 n-1}$ by a free action of the cyclic group $\mathbb{Z}_{m}$ (see [7, page 144). Then, we have an $m$-sheeted covering $\pi: S^{2 n-1} \longrightarrow L$. If $n>1$ and $m$ is odd, then for any 
vector bundle $\xi$ over $L$, the total Stiefel-Whitney class $w(\xi)=1$. In particular, $\operatorname{ucharrank}(L)=2 n-2$.

There are conditions under which one can obtain a natural upper bound on the upper characteristic rank of a space. One such condition is the existence of a spherical class. Recall that a cohomology class $x \in H^{k}\left(X ; \mathbb{Z}_{2}\right)$ is spherical if there exists a map $f: S^{k} \longrightarrow X$ with $f^{*}(x) \neq 0$. Note that a spherical class $x \in H^{k}\left(X ; \mathbb{Z}_{2}\right)$ is indecomposable as an element of the cohomology ring. We shall show that the upper characteristic rank of a space is bounded above by the degree of a spherical class in most cases.

Proposition 2.2. Let $X$ be a space and assume that $x \in H^{k}\left(X ; \mathbb{Z}_{2}\right)$ is spherical, $k \neq 1,2,4,8$. Then there does not exist a vector bundle $\xi$ over $X$ with $w_{k}(\xi)=x$ and we have $\operatorname{charrank}_{X}(\xi)<k$ for any $\xi$. As a consequence, for any covering $\pi: E \longrightarrow X$, we have ucharrank $(E)<k$ (in particular, ucharrank $(X)<k$ ).

Proof. Assume that $\xi$ is a vector bundle over $X$ with $w_{k}(\xi)=x \neq 0$. Let $f: S^{k} \longrightarrow X$ be a map with $f^{*}(x) \neq 0$. Then one has $w_{k}\left(f^{*} \xi\right)=f^{*}\left(w_{k}(\xi)\right) \neq 0$, which is impossible by Theorem 2.1. Hence there is no such $\xi$. Now since there is no $\xi$ with $w_{k}(\xi)=x$, and $x$ is indecomposable, we see that $\operatorname{charrank}_{X}(\xi)<k$ for any $\xi$. The rest of the claim follows from the fact that $f$ factors through the covering projection $\pi: E \longrightarrow X$. Indeed, we have $f=\pi \circ g$ for some $g: S^{k} \longrightarrow E$, and then $g^{*}\left(\pi^{*}(x)\right)=f^{*}(x) \neq 0$, which means that the class $\pi^{*}(x)$ is spherical. The proof is finished by taking $E$ in the role of $X$ in the preceding considerations.

When a spherical class has degree $k=1,2,4$, or 8 , there can exist vector bundles of characteristic rank greater than or equal to the degree of the spherical class. For example, the sphere $S^{k}$ with $k=1,2,4$, or 8 has upper characteristic rank equal to $k$. The complex projective space $\mathbb{C P}^{n}$ has a spherical class in degree 2, however ucharrank $\left(\mathbb{C P}^{n}\right)=2 n$ (see Example2). When a spherical class exists in degree 1,2,4 or 8 , we have the following observation:

Observation: Let $X$ be a space and assume that $x \in H^{k}\left(X ; \mathbb{Z}_{2}\right)$ is spherical, where $k=1,2,4,8$. Let $f: S^{k} \longrightarrow X$ be a map with $f^{*}(x) \neq 0$. Then for a vector bundle $\xi$ over $X$ with $\operatorname{charrank}_{X}(\xi) \geq k$, we can express $x$ as a polynomial $P\left(w_{1}(\xi), w_{2}(\xi), \ldots w_{k}(\xi)\right)$. But then $0 \neq f^{*}(x)=f^{*}\left(P\left(w_{1}(\xi), w_{2}(\xi), \ldots w_{k}(\xi)\right)\right)$ $=P\left(f^{*}\left(w_{1}(\xi)\right), f^{*}\left(w_{2}(\xi)\right), \ldots, f^{*}\left(w_{k}(\xi)\right)\right)$. Hence $f^{*}\left(w_{k}(\xi)\right) \neq 0$. Thus for any vector bundle $\xi$ over $X$ with $\operatorname{charrank}_{X}(\xi) \geq k$, we have $w_{k}(\xi) \neq 0$.

When $X$ is a connected closed smooth $d$-manifold, the characteristic rank, $\operatorname{charrank}_{X}(\xi)$, of $\xi$ takes values in a certain specific range. We prove the following.

Theorem 2.3. Let $X$ be a connected closed smooth d-manifold. Assume that $2 r_{X} \leq d$. Then, for any vector bundle $\xi$ over $X, \operatorname{charrank}_{X}(\xi)$ is either $d$ or less than $d-r_{X}$. 
Proof. Let $\xi$ be a vector bundle over $X$ with $\operatorname{charrank}_{X}(\xi) \geq d-r_{X}$. We shall show that $\operatorname{charrank}_{X}(\xi)=d$. Since, by Poincaré duality, the groups $H^{j}\left(X ; \mathbb{Z}_{2}\right)=0$ for $d-r_{X}<j<d$, the proof will be complete if the nonzero element in $H^{d}\left(X ; \mathbb{Z}_{2}\right)$ is a polynomial in the Stiefel-Whitney classes of $\xi$. As $\operatorname{charrank}_{X}(\xi) \geq d-r_{X} \geq r_{X}$, then by Lemma2.2. $H^{r_{X}}\left(X ; \mathbb{Z}_{2}\right) \cong \mathbb{Z}_{2}$. Hence $H^{d-r_{X}}\left(X ; \mathbb{Z}_{2}\right) \cong \mathbb{Z}_{2}$. Let $a, b, x$ denote the non-zero cohomology classes in degrees $r_{X}, d-r_{X}$ and $d$ respectively. The non-degeneracy of the pairing

$$
H^{r_{X}}\left(X ; \mathbb{Z}_{2}\right) \otimes H^{d-r_{X}}\left(X ; \mathbb{Z}_{2}\right) \longrightarrow H^{d}\left(X ; \mathbb{Z}_{2}\right)
$$

implies that $a \cdot b=x$. As $\operatorname{charrank}_{X}(\xi) \geq d-r_{X} \geq r_{X}$ we have, by Lemma2.1(1), $w_{r_{X}}(\xi) \neq 0$ and hence $w_{r_{X}}(\xi)=a$ and $b=p\left(w_{1}(\xi), w_{2}(\xi), \ldots\right)$ is a polynomial in the Stiefel-Whitney classes of $\xi$. This shows that

$$
x=w_{r_{X}}(\xi) \cdot p\left(w_{1}(\xi), w_{2}(\xi), \ldots\right)
$$

is a polynomial in the Stiefel-Whitney classes of $\xi$. This completes the proof of the theorem.

Let $X$ be a connected closed smooth $d$-manifold. If $X$ is an unoriented boundary, then any monomial in the Stiefel-Whitney classes of $X$ of total degree $d$ is zero (see [12, Theorem 4.9). Hence the non-zero element in $H^{d}\left(X ; \mathbb{Z}_{2}\right)$ is never a polynomial in the Stiefel-Whitney classes of $X$. We thus have the following corollary.

Corollary 2.3.1. Let $X$ be a connected closed smooth d-manifold. Assume that $2 r_{X} \leq d$. If $X$ is an unoriented boundary, then $\operatorname{charrank}(T X)<d-r_{X}$.

Remark 1. Balko and Korbaš [4] showed independently the following stronger version of Corollary 2.3.1 For any connected closed smooth $d$-dimensional manifold $X$ that is an unoriented boundary, if $s, s \leq \frac{d}{2}$, is (the biggest) such that $H^{s}\left(X ; \mathbb{Z}_{2}\right) \neq 0$, then $\operatorname{charrank}(X)<d-s$.

\section{Proof of Theorems 1.2, 1.3 and 1.4}

In this section we prove Theorems 1.2, 1.3, and 1.4. The proof of Theorem 1.2 is essentially the same as the proof of Theorem[1.1. We reproduce it here for completeness.

Proof of Theorem 1.2 Let $x=x_{1} \cdot x_{2} \cdots x_{s} \neq 0$ be a non-zero product of cohomology classes of positive degree and of maximal length. Then $x \in H^{d}\left(X ; \mathbb{Z}_{2}\right)$. If not, then by Poincaré duality one can find some $y$ in complementary dimension such that $x \cdot y \neq 0$ contradicting the maximality of $s$. By rearranging, we write

$$
x=\alpha_{1} \cdots \alpha_{m} \cdot \beta_{1} \cdots \beta_{n}
$$

where $\operatorname{deg}\left(\alpha_{i}\right) \leq k$ and $\operatorname{deg}\left(\beta_{j}\right) \geq k+1$. We note that $n \neq 0$. For otherwise the product $\alpha=\alpha_{1} \cdots \alpha_{m}$ which is now a polynomial in $w_{1}(\xi), \ldots, w_{k}(\xi)$, would be a 
non-zero element of total degree $d$ contradicting the assumption on $\xi$. Therefore, if $\beta=\beta_{1} \cdots \beta_{n}$, then $\operatorname{deg}(\beta) \geq k+1$. Thus $\operatorname{deg}(\alpha) \leq d-(k+1)$. Thus

$$
\begin{aligned}
\operatorname{cup}(X) & =m+n \\
& \leq \frac{\operatorname{deg}(\alpha)}{r_{X}}+\frac{\operatorname{deg}(\beta)}{(k+1)} \\
& =\frac{\operatorname{deg}(\alpha)}{r_{X}}+\frac{(d-\operatorname{deg}(\alpha))}{(k+1))} \\
& =\frac{\left(\left(k+1-r_{X}\right) \operatorname{deg}(\alpha)+d r_{X}\right)}{r_{X}(k+1)} \\
& \leq \frac{\left(\left(k+1-r_{X}\right)(d-(k+1))+d r_{X}\right)}{r_{X}(k+1)} \\
& =1+\frac{d-k-1}{r_{X}} .
\end{aligned}
$$

This completes the proof.

Proof of Theorem 1.3. Let $\xi$ be any vector bundle over $X$ with

$$
\operatorname{charrank}_{X}(\xi)=\operatorname{ucharrank}(X)=\operatorname{dim}(X) .
$$

Let $\operatorname{cup}(X)=k$. We shall show that some product of the Stiefel-Whitney classes of $\xi$ of length $k$ is non-zero. Let

$$
x=x_{1} \cdot x_{2} \cdots x_{k} \neq 0
$$

be a non-zero product of cohomology classes $x_{i} \in H^{*}\left(X ; \mathbb{Z}_{2}\right)$ with $\operatorname{deg}\left(x_{i}\right) \geq 1$. As $\operatorname{charrank}_{X}(\xi)=\operatorname{dim}(X)$, each $x_{i}$ can be written as a sum of monomials in the Stiefel-Whitney classes of $\xi$. Thus $x$ can be written as a sum of monomials in the Stiefel-Whitney classes of $\xi$, each of length at least $k$. Note that the monomials of length greater than $k$ are zero by hypothesis. As $x \neq 0$, it follows that some monomial in the Stiefel-Whitney classes of $\xi$ of length $k$ is non-zero. This completes the proof of the theorem.

Remark 2. (1) The proof of Theorem 1.3 actually shows that if some product $x=x_{1} \cdots x_{t} \neq 0$ with $1 \leq \operatorname{deg}\left(x_{i}\right) \leq \ell$, then for any vector bundle $\xi$ over $X$ with charrank ${ }_{X}(\xi) \geq \ell$ some product of the Stiefel-Whitney classes of $\xi$ of length greater than or equal to $t$ is non-zero.

(2) The conclusion of Theorem 1.3 is not true if ucharrank $(X)<\operatorname{dim}(X)$. If $X=S^{k}, k \neq 1,2,4,8$, then $\operatorname{ucharrank}(X)=k-1<k, \operatorname{cup}(X)=1$ however $w(\xi)=1$ for any vector bundle $\xi$ over $X$.

Proof of Theorem1.4, First note that the assumption ucharrank $(X) \geq 1$ is odd clearly implies that the function

$$
f: \operatorname{Vect}_{\mathbb{R}}(X) \longrightarrow \mathbb{Z}_{2}
$$


defined by

$$
f(\xi)=\operatorname{charrank}_{X}(\xi)(\bmod 2)
$$

is surjective. We shall now check that $f$ is a semi-group homomorphism. To see this, let $\xi$ and $\eta$ be two bundles over $X$. We have the following cases.

If $\xi$ and $\eta$ are both orientable, then so is $\xi \oplus \eta$. Hence $w_{1}(\xi \oplus \eta)=0$. As $r_{X}=1$, it follows that $\operatorname{charrank}_{X}(\xi \oplus \eta)=0$. The same argument shows that $\operatorname{charrank}_{X}(\xi)=0=\operatorname{charrank}_{X}(\eta)$. Thus in this case we have $f(\xi \oplus \eta)=$ $f(\xi)+f(\eta)$.

Next suppose that both $\xi$ and $\eta$ are non-orientable. Then, on the one hand, $\xi \oplus \eta$ is orientable and hence $f(\xi \oplus \eta)=0$ as $r_{X}=1$. On the other hand, as $\xi$ and $\eta$ are non-orientable, we have

$$
f(\xi)=1=f(\eta)
$$

Thus, we have the equality $f(\xi \oplus \eta)=f(\xi)+f(\eta)$.

Finally, assume that $\xi$ is orientable and $\eta$ is not. Then $\xi \oplus \eta$ is not orientable and hence $f(\xi \oplus \eta)=1, f(\xi)=0$ and $f(\eta)=1$. So in this case we have $f(\xi \oplus \eta)=$ $f(\xi)+f(\eta)$. This completes the proof that $f$ is a semi-group homomorphism.

This gives rise to a surjective homomorphism

$$
\tilde{f}: K O(X) \longrightarrow \mathbb{Z}_{2}
$$

defined by $\widetilde{f}(\xi-\eta)=f(\xi)-f(\eta)$. It is now clear that $\tilde{f}$ is zero on the $\mathbb{Z}$ summand of $K O(X)=\mathbb{Z} \oplus \widetilde{K O}(X)$ and restricts to an epimorphism $\tilde{f}: \widetilde{K O}(X) \longrightarrow \mathbb{Z}_{2}$. This completes the proof.

\section{Computations and examples}

In this section we give a proof of Theorem 1.5 and compute the characteristic rank of vector bundles over $X$, where $X$ is one of the following: the product of spheres $S^{d} \times S^{m}$, the real or complex projective space, the product space $S^{1} \times \mathbb{C P}^{n}$, the Moore space $M\left(\mathbb{Z}_{2}, n\right)$ and the stunted projective space $\mathbb{R} \mathbb{P}^{n} / \mathbb{R P}^{m}$.

We begin by describing the characteristic rank of vector bundles over $X=$ $S^{d} \times S^{m}$. First note that if $d=m$, then as $r_{X}=d$ and $\operatorname{dim}_{\mathbb{Z}_{2}} H^{d}\left(X ; \mathbb{Z}_{2}\right)=2$, it follows from Lemma2.2 (1) that $\operatorname{ucharrank}(X)=r_{X}-1=d-1$.

Lemma 4.1. Let $X=S^{d} \times S^{m}$ with $d<m$. Then,

$$
\operatorname{ucharrank}(X)=\left\{\begin{array}{cl}
d-1 & \text { if } d \neq 1,2,4,8, \\
m-1 & \text { if } d=1,2,4,8, m \neq 2,4,8 \\
d+m & \text { if } d, m=1,2,4,8
\end{array}\right.
$$

Proof. The lemma follows from the observations made after Theorem 2.1. We note that $r_{X}=d$ and consider the maps

$$
S^{d} \stackrel{i}{\longrightarrow} S^{d} \times S^{m} \stackrel{\pi_{1}}{\longrightarrow} S^{d},
$$




$$
S^{m} \stackrel{j}{\longrightarrow} S^{d} \times S^{m} \stackrel{\pi_{2}}{\longrightarrow} S^{m},
$$

where $i$ is the map $x \mapsto(x, y)$ for a fixed $y \in S^{m}$ and $\pi_{1}$ and $\pi_{2}$ are projections onto the the first and second factors. The map $j$ is similarly defined. The homomorphisms $i^{*}$ and $j^{*}$ are isomorphisms (with inverses $\pi_{1}^{*}$ and $\pi_{2}^{*}$ respectively) in degree $d$ and $m$ respectively.

Assume that $d \neq 1,2,4,8$ and let $\xi$ be a vector bundle over $X$. Then as $w_{d}\left(i^{*} \xi\right)=0$, it follows that $w_{d}(\xi)=0$. Thus by Lemma2.1 (1) we have $\operatorname{charrank}_{X}(\xi)=r_{X}-1=d-1$.

Next assume that $d=1,2,4,8$ and $m \neq 2,4,8$. Let $\nu_{d}$ denote the Hopf bundle over $S^{d}$. As $w_{d}\left(\nu_{d}\right) \neq 0$, it follows that $w_{d}\left(\pi_{1}^{*} \nu_{d}\right) \neq 0$. Thus $\operatorname{charrank}_{\pi_{1}^{*} \nu_{d}}(X) \geq$ $m-1$. Since $m \neq 1,2,4,8$, for any vector bundle $\xi$ over $X$ we must have $w_{m}(\xi)=0$. This completes the proof that $\operatorname{charrank}_{\pi_{1}^{*} \nu_{d}}(X)=m-1$ and that $\operatorname{ucharrank}(X)=m-1$.

Finally, let $d=1,2,4,8$ and $m=1,2,4,8$. Let $\nu_{d}$ and $\nu_{m}$ denote the Hopf bundles over $S^{d}$ and $S^{m}$ respectively. Then, clearly $w_{d}\left(\pi_{1}^{*} \nu_{d} \oplus \pi_{2}^{*} \nu_{m}\right) \neq 0$, $w_{m}\left(\pi_{1}^{*} \nu_{d} \oplus \pi_{2}^{*} \nu_{m}\right) \neq 0$ and $w_{d+m}\left(\pi_{1}^{*} \nu_{d} \oplus \pi_{2}^{*} \nu_{m}\right) \neq 0$. This shows that in this case charrank $(X)=d+m$. This completes the proof of the lemma.

We now come to the proof of Theorem 1.5. First recall that the Dold manifold $P(m, n)$ is an $(m+2 n)$-dimensional manifold defined as the quotient of $S^{m} \times \mathbb{C P}^{n}$ by the fixed point free involution $(x, z) \mapsto(-x, \bar{z})$. This gives rise to a two-fold covering

$$
\mathbb{Z}_{2} \hookrightarrow S^{m} \times \mathbb{C P}^{n} \longrightarrow P(m, n),
$$

and via the projection $S^{m} \times \mathbb{C P}^{n} \longrightarrow S^{m}$, a fiber bundle

$$
\mathbb{C P}^{n} \hookrightarrow P(m, n) \longrightarrow \mathbb{R} \mathbb{P}^{m}
$$

with fiber $\mathbb{C P}^{n}$ and structure group $\mathbb{Z}_{2}$. In particular, for $n=1$, we have a fiber bundle

$$
S^{2} \hookrightarrow P(m, 1) \longrightarrow \mathbb{R} \mathbb{P}^{m}
$$

The $\mathbb{Z}_{2}$-cohomology ring of $P(m, n)$ is given by [6]

$$
H^{*}\left(P(m, n) ; \mathbb{Z}_{2}\right)=\mathbb{Z}_{2}[c, d] /\left(c^{m+1}, d^{n+1}\right)
$$

where $c \in H^{1}\left(P(m, n) ; \mathbb{Z}_{2}\right)$ and $d \in H^{2}\left(P(m, n) ; \mathbb{Z}_{2}\right)$.

We shall make use of the following result which shows the existence of certain bundles with suitable Stiefel-Whitney classes.

Proposition 4.1. ([13], page 86) Over $P(m, n)$,

(1) there exists a line bundle $\xi$ with total Stiefel-Whitney class $w(\xi)=1+c$;

(2) there exists a 2-plane bundle $\eta$ with total Stiefel-Whitney class $w(\eta)=$ $1+c+d$. 
Proof of Theorem 1.5, Let $X=\mathbb{R}^{n}$ be the real projective space. Then $r_{X}=1$. Let $\xi$ be a vector bundle over $X$. If $\xi$ is orientable, then $w_{1}(\xi)=0$ and hence, by Lemma2.1 (1), $\operatorname{charrank}_{X}(\xi)=0$. On the other hand if $\xi$ is non-orientable, then $w_{1}(\xi) \neq 0$ and hence $\operatorname{charrank}_{X}(\xi)=n$ as $H^{*}\left(X ; \mathbb{Z}_{2}\right)$ is polynomially generated by the non-zero element in $H^{1}\left(X ; \mathbb{Z}_{2}\right)$. This proves $(1)$.

To prove (2), let $X=S^{1} \times \mathbb{C P}^{n}$, then $r_{X}=1$. The $\mathbb{Z}_{2}$-cohomology ring of $X$ is given by

$$
H^{*}\left(X ; \mathbb{Z}_{2}\right)=H^{*}\left(S^{1} ; \mathbb{Z}_{2}\right) \otimes H^{*}\left(\mathbb{C P}^{n} ; \mathbb{Z}_{2}\right) \cong \mathbb{Z}_{2}[a, b] /\left(a^{2}, b^{n+1}\right),
$$

where $a$ is of degree one and $b$ is of degree two. Let $\xi$ be a vector bundle over $X$. Evidently, $\operatorname{charrank}_{X}(\xi)$ is completely determined by the first two StiefelWhitney classes of $\xi$.

We look at several cases. If $w_{1}(\xi)$ and $w_{2}(\xi)$ are both non-zero, then the description of the cohomology ring $H^{*}\left(X ; \mathbb{Z}_{2}\right)$ forces $\operatorname{charrank}_{X}(\xi)=2 n+1$. If $w_{1}(\xi)=0$, we have $\operatorname{charrank}_{X}(\xi)=0$. If $w_{1}(\xi) \neq 0$ and $w_{2}(\xi)=0$, then $\operatorname{charrank}_{X}(\xi)=1$. This completes the proof of $(2)$.

Finally, the proof of (3) is similar to the case (2) above in view of Proposition 4.1. Indeed, if $w_{1}(\eta)=c \neq 0$ and $w_{2}(\eta)=d \neq 0$ (there exists such an $\eta$; see Proposition 4.1), then we have $\operatorname{charrank}_{X}(\eta)=2 n+m$. If $w_{1}(\xi)=c \neq 0$ and $w_{2}(\xi)=0$ (there exists such a $\xi$; see Proposition 4.2), we have $\operatorname{charrank}_{X}(\xi)=1$, as $c^{2} \neq d$. For other possible vector bundles, the situation is clear. This completes the proof of (3) and the theorem.

Remark 3. (1) We remark that, in the case (2) of the theorem above, there exists a line bundle $\gamma$ over $X$ such that $w_{1}(\gamma) \neq 0$. Thus, $\operatorname{charrank}_{X}(\gamma)=1$. We also can find a 2-plane bundle $\eta$ over $X$ such that $w_{1}(\eta)=0$ and $w_{2}(\eta) \neq 0$. $\operatorname{Thus}_{\operatorname{charrank}}(\eta)=0$. Then for the Whitney sum $\gamma \oplus \eta$ we have $w_{1}(\gamma \oplus \eta)=$ $w_{1}(\gamma) \neq 0$ and $w_{2}(\gamma \oplus \eta)=w_{2}(\eta) \neq 0$ and hence $\operatorname{charrank}_{\gamma \oplus \eta}(X)=2 n+1$. The bundles $\gamma$ and $\eta$ can be obtained as the pull backs of suitable canonical bundles over $S^{1}=\mathbb{R P}^{1}$ and $\mathbb{C P}^{n}$ via the projections. Thus, over $X=S^{1} \times \mathbb{C P}^{n}$, there exist vector bundles having all the three possible characteristic ranks.

(2) The function $f: \operatorname{Vect}_{\mathbb{R}}(X) \longrightarrow \mathbb{Z}_{2}$ constructed in the proof of Theorem 1.4 is in general not a semi-ring homomorphism. For example, let $\gamma$ denote the canonical line bundle over $X=\mathbb{R P}^{n}$ ( $n$ odd). Then $w_{1}(\gamma) \neq 0$ and hence $f(\gamma)=1 \in \mathbb{Z}_{2}$. Now, as $\gamma \otimes \gamma$ is a trivial bundle, we have $w_{1}(\gamma \otimes \gamma)=0$ and therefore, $f(\gamma \otimes \gamma)=0 \in \mathbb{Z}_{2}$. Clearly, $0=f(\gamma \otimes \gamma) \neq f(\gamma) \cdot f(\gamma)=1$.

Example 2. Let $X=\mathbb{C P}^{n}$ be the complex projective space. Then $r_{X}=2$. Let $\xi$ be a vector bundle over $X$. Then $\operatorname{charrank}_{X}(\xi)=1$ if $w_{2}(\xi)=0$ and $\operatorname{charrank}_{X}(\xi)=2 n$ if $w_{2}(X) \neq 0$. For the canonical (complex) line bundle $\gamma$ over $X$ we have $\operatorname{charrank}_{X}(\gamma)=2 n$.

We now give some examples where the bound for the cup length given by Theorem 1.2 is sharper than that given by Theorem 1.1 
Example 3. Let $X=S^{2} \times S^{6}$ and let $\pi_{1}: X \longrightarrow S^{2}$ be the projection. Let, as usual, $\nu_{2}$ denote the Hopf bundle over $S^{2}$. Then, $\operatorname{charrank}_{T X}(X)=1$, and $\operatorname{charrank}_{X}(\xi)=5$ where $\xi=\pi_{1}^{*} \nu_{2}$. The bundle $\xi$ satisfies the condition of Theorem 1.2 with $k=5$. Then the bound for the cup length, $\operatorname{cup}(X)$, of $X$ given by Theorem 1.1 is 4 and that given by Theorem 1.2 is 2 .

Example 4. Let $X=S^{4} \times S^{8}$. Let $\xi=\pi_{1}^{*} \nu_{4} \oplus \pi_{2}^{*} \nu_{8}$. Then, $\operatorname{charrank}_{T X}(X)=3$ and $\operatorname{charrank}_{X}(\xi)=12$. Then $\xi$ satisfies the condition of Theorem 1.2 with $k=7$. Then the bound for the cup length, $\operatorname{cup}(X)$, of $X$ given by Theorem 1.1 is 3 and that given by Theorem 1.2 is 2 .

Remark 4. These sharper estimates of Examples 3 and 4 can also be obtained from Theorem A [9].

We now compute $\operatorname{charrank}_{X}(\xi)$ where $X$ is the Moore space $M\left(\mathbb{Z}_{2}, n\right), n>1$, and $\xi$ a vector bundle over $X$. We recall that $X$ is an $(n-1)$-connected $(n+1)$ dimensional $C W$-complex. Note that $M\left(\mathbb{Z}_{2}, 1\right)$ is the real projective space $\mathbb{R P}^{2}$ and $M\left(\mathbb{Z}_{2}, n\right)$ is the iterated suspension $\Sigma^{n} M\left(\mathbb{Z}_{2}, 1\right)$. We refer to [7] for basic properties of Moore spaces. We prove the following.

Proposition 4.2. Let $X$ denote the Moore space $M\left(\mathbb{Z}_{2}, n\right)$ with $n>1$. Then,

$$
\operatorname{ucharrank}(X)=\left\{\begin{array}{cc}
n-1 & \text { if } n \neq 2 \\
3 & \text { if } n=2
\end{array}\right.
$$

Proof. The Moore space $X$ is an $(n+1)$-dimensional $C W$-complex with $n$ skeleton $S^{n}$. Let $i: S^{n} \hookrightarrow X$ denote the inclusion map. Using the cellular chain complex, for example, it is easy to see that the homomorphism

$$
i^{*}: H^{n}\left(X ; \mathbb{Z}_{2}\right) \longrightarrow H^{n}\left(S^{n} ; \mathbb{Z}_{2}\right)
$$

in degree $n$ is an isomorphism and hence the non-zero element in $H^{n}\left(X ; \mathbb{Z}_{2}\right)$ is spherical.

Assume that $n \neq 2,4,8$. Since $X$ is $(n-1)$-connected it follows from Proposition 2.2 that $\operatorname{charrank}_{X}(\xi)=n-1$ for any $\xi$ over $X$. This proves the first equality for $n \neq 2,4,8$.

Next, for $X=M\left(\mathbb{Z}_{2}, n\right)$, we observe that there is a cofiber sequence

$$
S^{n} \stackrel{f}{\longrightarrow} S^{n} \longrightarrow X \longrightarrow S^{n+1} \longrightarrow S^{n+1}
$$

where $f$ is a degree 2 map. This gives rise to an exact sequence

$$
\widetilde{K O}\left(S^{n+1}\right) \longrightarrow \widetilde{K O}(X) \longrightarrow \widetilde{K O}\left(S^{n}\right) \stackrel{f^{*}}{\longrightarrow} \widetilde{K O}\left(S^{n}\right) .
$$

When $n=4,8$ the homomorphism $f^{*}$ is injective and hence the homomorphism $\widehat{K O}\left(S^{n+1}\right) \longrightarrow \widehat{K O}(X)$ is surjective. When $n=2$, the homomorphism $f^{*}$ is the zero homomorphism and hence the homomorphism $\widetilde{K O}(X) \longrightarrow \widetilde{K O}\left(S^{n}\right)$ 
is surjective. These obeservations follow from the fact that $\widetilde{K O}\left(S^{4}\right)=\mathbb{Z}=$ $\widetilde{K O}\left(S^{8}\right)$ and $\widetilde{K O}\left(S^{2}\right)=\mathbb{Z}_{2}$ together with the fact that $f$ is a degree 2 map.

Thus when $n=4,8$ we have by Theorem 2.1 that $w(\xi)=1$ for any vector bundle over $X=M\left(\mathbb{Z}_{2}, n\right)$. This completes the proof of the first equality when $n=4,8$.

Finally let $X=M\left(\mathbb{Z}_{2}, 2\right)$. Then $X$ is a simply connected 3-dimensional $C W$ complex. We shall show that there exists a bundle $\xi$ over $X$ with $w_{2}(\xi) \neq$ 0 and $w_{3}(\xi) \neq 0$. As the homomorphism $\widetilde{K O}(X) \longrightarrow \widetilde{K O}\left(S^{2}\right)$ is surjective and $w_{2}\left(\nu_{2}\right) \neq 0$, there exists a bundle $\xi$ over $X$ with $w_{2}(\xi) \neq 0$. For this vector bundle $\xi$ over $X$ the Stiefel-Whitney class $w_{3}(\xi) \neq 0$. To see this we observe that if $a \in H^{1}\left(\mathbb{R P}^{2} ; \mathbb{Z}_{2}\right)=H^{1}\left(M\left(\mathbb{Z}_{2}, 1\right) ; \mathbb{Z}_{2}\right)$ is the unique non-zero element, then $S q^{1}(a)=a^{2} \neq 0$. Thus, by Wu's formula and the fact that the Steenrod squares commute with the suspension homomorphism we see that $S q^{1}\left(w_{2}(\xi)\right)=w_{1}(\xi) w_{2}(\xi)+w_{3}(\xi)=w_{3}(\xi) \neq 0$. This completes the proof of the second equality.

Proposition 4.3. Let $X$ denote the stunted projective space $\mathbb{R} \mathbb{P}^{n} / \mathbb{R} \mathbb{P}^{m}$ with $1 \leq m \leq n-2$. Then

$$
\operatorname{ucharrank}(X)=\left\{\begin{array}{cl}
m & \text { if } m+1 \neq 2,4,8 \\
m+1 & \text { if } m+1=2,4,8
\end{array}\right.
$$

Proof. The stunted projective space $X$ is $m$-connected with $(m+1)$-skeleton $X^{(m+1)}=S^{m+1}$. If $f: S^{m+1}=X^{(m+1)} \longrightarrow X$ denotes the inclusion map, then it is easy to check that the homomorphism

$$
f^{*}: H^{m+1}\left(X ; \mathbb{Z}_{2}\right) \longrightarrow H^{m+1}\left(S^{m+1} ; \mathbb{Z}_{2}\right)
$$

is an isomorphism. Thus, the non-zero element in $H^{m+1}\left(X ; \mathbb{Z}_{2}\right)$ is spherical. The first equality of the proposition now follows from Proposition 2.2 .

Let $X=\mathbb{R} \mathbb{P}^{n} / \mathbb{R P}^{m}$ with $m+1=2,4,8$. It is clear that the inclusion map

$$
\mathbb{R} \mathbb{P}^{m+2} / \mathbb{R} \mathbb{P}^{m} \longrightarrow \mathbb{R P}^{n} / \mathbb{R} \mathbb{P}^{m}
$$

where $n \geq m+2$ induces isomorphism in $\mathbb{Z}_{2}$-cohomology in degree $i$ for all $i \leq m+2$. Since $(m+2)$ is odd we have a splitting

$$
\mathbb{R} \mathbb{P}^{m+2} / \mathbb{R} \mathbb{P}^{m}=S^{m+2} \vee S^{m+1} \text {. }
$$

It follows that $X$ has a spherical class in degree $(m+2)$ and hence by Proposition 2.2 we have ucharrank $(X) \leq m+1$. We shall prove the equality by showing that there exists a bundle $\xi$ over $X$ with $w_{m+1}(\xi) \neq 0$.

As $\mathbb{R} \mathbb{P}^{m+2} / \mathbb{R P}^{m}=S^{m+1} \vee S^{m+2}$, the Hopf bundle $\nu_{m+1}$ over $S^{m+1}$ extends over $S^{m+1} \vee S^{m+2}$ to give a vector bundle $\xi$ with $w_{m+1}(\xi) \neq 0$. It is well known [1] that for any $n \geq m+2$ the inclusion map

$$
\mathbb{R} \mathbb{P}^{m+2} / \mathbb{R} \mathbb{P}^{m} \hookrightarrow \mathbb{R} \mathbb{P}^{n} / \mathbb{R P}^{m}
$$


induces an epimorphism in reduced $K O$-groups. Thus there is a vector bundle over $\mathbb{R} \mathbb{P}^{n} / \mathbb{R P}^{m}$ with the required property.

Acknowledgement. We are indebted to Professor J. Korbaš for his detailed and helpful comments on an earlier draft of this manuscript. In particular, we thank him for showing us the proof of Corollary 2.1.1. The original statement of the corollary only contained the conclusion that ucharrank $(X)<d$, under the assumption that $X$ is orientable and $d \neq 1,2,4,8$. We also thank him for sending us a copy of his paper [10. We would like to thank the anonymous referee for his detailed suggestions. In particular, we thank him for showing us the proof of Proposition 4.2. This is shorter and stronger than proof given by the authors.

\section{REFERENCES}

[1] Adams, J. F.: Vector fields on Spheres, Ann. Math. 75 (1962), 603-632.

[2] Atiyah, M.-Hirzebruch, F.: Bott periodicity and the parallelizability of the spheres, In: Proc. Cambridge Philos. Soc., 57 (1961), pp. 223-226.

[3] Balko, L.-Korbaš, J.: A note on the characteristic rank of a smooth manifold, Group actions and homogeneous spaces, Fak. Mat. Fyziky Inform. Univ. Komenského, Bratislava, 2010, pp. 1-8.

[4] Balko, L'-Korbaš, J.: A note of the characteristic rank of null-cobordant manifolds, To appear in Acta. Math. Hungar, 2011/2012.

[5] Borel, A.: Sur la cohomologie des espaces fibrés principaux et des espaces homogènes de groupes de Lie compacts, Ann. of Math. 57 (1953), 115-207.

[6] Dold, A.: Erzeugende der Thomschen Algebra N, Math. Z. 65 (1956), 25-35.

[7] Hatcher, A.: Algebraic Topology, Cambridge Univ. Press, 2002.

[8] Husemoller, D.: Fibre Bundles, Springer-Verlag, New York, 1966.

[9] Korbaš, J.: Bounds for the cup-length of Poincaré spaces and their applications, Topology Appl. 153 (2006), 2976-2986.

[10] Korbaš, J.: The cup-length of the oriented Grassmannians vs a new bound for zero cobordant manifolds, Bull. Belg. Math. Soc.-Simon Stevin 17 (2010), 69-81.

[11] Milnor, J.: Some consequences of a theorem of Bott, Ann. of Math. 68 (1958), 444-449.

[12] Milnor, J.-Stasheff, J.: Characteristic Classes, Princeton Univ. Press, Princeton, 1974.

[13] Stong, R. E.: Vector bundles over Dold manifolds, Fund. Math. 169 (2001), 85-95.

Stat-Math UNit

Indian Statistical Institute

8Th Mile, Mysore Road, RVCE Post

BANGALORE 560059

INDIA.

E-mail address: ani@isibang.ac.in, thakur@isibang.ac.in 